\title{
MAGNETO-ELASTIC INTERNAL RESONANCE OF AN AXIALLY MOVING CONDUCTIVE BEAM IN THE MAGNETIC FIELD
}

\author{
JIE WANG \\ Key Laboratory of Mechanical Reliability for Heavy Equipments and Large Structures of Hebei Province, \\ Yanshan University, Qinhuangdao, P.R. China and \\ Department of Mechanics, School of Aerospace Engineering, Beijing Institute of Technology, Beijing, P.R. China \\ YUDA HU \\ Key Laboratory of Mechanical Reliability for Heavy Equipments and Large Structures of Hebei Province, \\ Yanshan University, Qinhuangdao, P.R. China; e-mail: huyuda03@163.com \\ Yu Su \\ Department of Mechanics, School of Aerospace Engineering, Beijing Institute of Technology, Beijing, P.R. China \\ LIANGFEI GONG \\ State Key Laboratory of Explosion Science and Technology, Beijing Institute of Technology, Beijing, P.R. China \\ QINGNAN ZHANG \\ Department of Mechanics, School of Aerospace Engineering, Beijing Institute of Technology, Beijing, P.R. China
}

The Hamiltonian principle is applied to the nonlinear vibration equation of an axially moving conductive beam in the magnetic field with consideration of the axial velocity, axial tension, electromagnetic coupling effect and complex boundary conditions. Nonlinear vibration characteristics of the free vibrating beam under 1:3 internal resonances are studied based on our approach. For beams with one end fixed and the other simply supported, the nonlinear vibration equation is dispersed by the Galerkin method, and the vibration equations are solved by the multiple-scales method. As a result, the coupled relations between the first-order and second-order vibration modes are obtained in the internal resonance system. Firstly, the influence of initial conditions, axial velocity and the external magnetic field strength on the vibration modes is analysed in detail. Secondly, direct numerical calculation on the vibration equations is carried out in order to evaluate the accuracy of the perturbation approach. It is found that through numerical calculations, in the undamped system, the vibration modes are more sensitive to the initial value of vibration amplitude. The amplitude changes of the first-order and second-order modes resulting from the increase of the initial amplitude value of the vibration modes respectively are very special, and present a "reversal behaviour". Lastly, in the damped system, the vibration modes exhibit a trend of coupling attenuation with time. Its decay rate increases when the applied magnetic field strength becomes stronger.

Keywords: magneto-elastic, conductive beam, internal resonance, axially moving, multiple scales

\section{Introduction}

Axial motion structures and devices are widely used in the the engineering in form of magnetically levitated trains, electromotors, and telphers. When such components work in an environment with an electromagnetic field there appears a number of multi-physics coupling effects such as force, electricity and magnetism, which affect safety and reliability of the system. A great number of in-depth theoretical studies have been carried out on such traditional problems of axially moving beams, plates and strings. For an axial motion system, Chen et al. (2010) and Ding and 
Chen (2010) studied nonlinear forced vibration of an axially moving viscoelastic beam. As a result, the influence from the axial velocity and boundary conditions on the structure vibration frequency and dynamic stability was analyzed. Hu et al. (2015) established a coupled nonlinear magneto-elastic vibration equation of the axial motion of a conducting plate, and investigated nonlinear vibration and chaotic motion of the plate. Pellicano (2005) obtained the complex dynamic response of an axial motion system under the external excitation load from a needle. Based on the generalized integral transform technique (GITT), Yan et al. (2015) investigated nonlinear dynamic behavior in the transverse vibration of an axially accelerating viscoelastic Timoshenko beam with an external harmonic excitation. Sahoo et al. $(2015,2016)$ analyzed the nonlinear transverse vibration of an axially moving beam subject to two-frequency excitation. Analytical and numerical approach was applied to find the steady-state and dynamic behavior of an axially accelerating viscoelastic beam subject to two-frequency parametric excitation in the presence of internal resonance. Pratiher and Dwivedy (2009) and Pratiher (2011) studied non-linear dynamics of a soft magneto-elastic Cartesian manipulator with a large transverse deflection. In addition, the non-linear response of a magneto-elastic translating beam having a prismatic joint for higher resonance conditions was studied by them. Wu (2007) investigated dynamic instability of a pinned beam subjected to an alternating magnetic field and thermal load with nonlinear strain, and made of a physically nonlinear thermoplastic material. Applying Hamilton's principle, the equation of motion with a damping factor, the induced current and thermal load was derived. Wang et al. (2011) proposed an $\mathrm{H}$ method for vibration control of an iron cantilever beam with axial velocity by applying a non-contact force through permanent magnets. For the internal resonance, Li et al. (2017) investigated magneto-elastic internal resonances of a rectangular conductive thin plate with different size ratios. They obtained amplitude-frequency response equations of 1:3 internal resonances by Galerkin and multi-scale methods. Mao et al. (2016a,b) firstly studied the forced vibration response of a pipe conveying fluid and super-harmonic resonances of a super-critical axially moving beam, with 3:1 internal resonance. Parametric and 3:1 internal resonance of axially moving viscoelastic beams on elastic foundation was analytically and numerically investigated by Tang et al. (2016).

In this paper, the internal resonance of an axially moving conductive beam in the magnetic field is investigated. The magnetic elastic vibration equation is to be obtained for the axially moving beam in a magnetic field, and 1:3 internal resonances are to be analyzed as well.

\section{The vibration equation}

The mechanical model of an axially moving conductive beam in a constant magnetic field is shown in Fig. 1. The magnetic field intensity is described by $\mathbf{B}_{0}\left(0, B_{0 y}, 0\right)$; where $l, h$ and $b$ denote length, height and width of the beam, respectively; the axial speed is $c$.

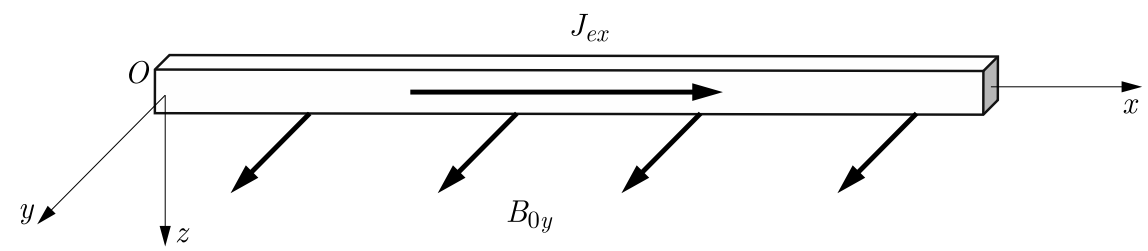

Fig. 1. An axially moving conducting elastic beam in a magnetic field. The letters $x, y$ and $z$ represent cartesian coordinates, and $J_{e x}$ denotes the $x$-axis component of the induced current density caused by the moving beam in an external magnetic field

The magneto-elastic vibration equation of the axially moving current carrying beam in the transverse magnetic field, shown as Eq. (2.1), is derived from the Hamilton variational principle. 
Interested readers are referred to the work of Hu and Wang (2017) for the derivation and origin of this vibration equation

$$
\begin{aligned}
\rho A & \frac{\partial^{2} w}{\partial t^{2}}+2 \rho A c \frac{\partial^{2} w}{\partial x \partial t}+\left[\rho A c^{2}-F_{0 x}-\frac{3}{2} E A\left(\frac{\partial w}{\partial x}\right)^{2}\right] \frac{\partial^{2} w}{\partial x^{2}}+\sigma_{0} A B_{0 y}^{2} \frac{\partial w}{\partial t} \\
& +E I \frac{\partial^{4} w}{\partial x^{4}}+\sigma_{0} c A B_{0 y}^{2} \frac{\partial w}{\partial x}=0
\end{aligned}
$$

where $w(x, t)$ is the transverse displacement of the beam. $E, \rho, \sigma_{0}$ denote Young's modulus, material density and conductivity, respectively. $F_{0 x}$ is the axial tension, and $I$ is the area moment of inertia. The rectangular cross section is $A=b \times h . t$ is the time variable.

\section{Magneto-elastic internal resonance}

For the one end fixed and the other simply supported boundary condition, the expression is

$$
\left.w\right|_{x=0}=\left.0 \quad \frac{\partial w}{\partial x}\right|_{x=0}=0 \quad \text { and }\left.\quad w\right|_{x=l}=\left.0 \quad \frac{\partial^{2} w}{\partial x^{2}}\right|_{x=l}=0
$$

The assumed displacement solution satisfying the particular boundary condition is described in the following form

$$
w=\sum_{n=1}^{2} Q_{n}(t) X_{n}(x)
$$

where $Q(t)$ denotes the amplitude of the mode, and the function $X_{n}(x)$ is determined by

$$
\begin{aligned}
& X_{n}(x)=\cosh p_{n} x-\cos p_{n} x-\varsigma_{n}\left(\sinh p_{n} x-\sin p_{n} x\right) \\
& \varsigma_{n}=\frac{\cosh p_{n} l+\cos p_{n} l}{\sinh p_{n} l+\sin p_{n} l} \quad p_{n}=\frac{(4 n+1) \pi}{4 l}
\end{aligned}
$$

When Eq. (3.2) is substituted into Eq. (2.1), the vibration differential equations of the axially moving beam in the magnetic field are derived by the Galerkin method

$$
\begin{aligned}
\rho A & \sum_{n=1}^{2} A_{n i} \ddot{Q}_{n}(t)+\sum_{n=1}^{2}\left(c A \sigma_{0} B_{0 y}^{2} B_{n i}+\rho A c^{2} C_{n i}-F_{0 x} C_{n i}+E I D_{n i}\right) Q_{n}(t) \\
& +\sum_{n=1}^{2}\left(2 \rho A c B_{n i}+\sigma_{0} A B_{0 y}^{2} A_{n i}\right) \dot{Q}_{n}(t)-\frac{3}{2} E A\left(S_{1 i} Q_{1}^{3}+S_{2 i} Q_{2}^{3}+S_{3 i} Q_{1} Q_{2}^{2}+S_{4 i} Q_{1}^{2} Q_{2}\right)=0
\end{aligned}
$$

where $i=1,2$ and the coefficients are provided in Appendix I.

After the coefficients of Eq. (3.3) are simplified, the differential equations of transverse vibration of the beam can be obtained by the dimensionless method

$$
\begin{aligned}
& \ddot{q}_{1}(\tau)+g_{1}^{2} q_{1}(\tau)=-\varepsilon \mu_{11} \dot{q}_{1}(\tau)-\varepsilon \mu_{12} \dot{q}_{2}(\tau)+\varepsilon\left(s_{11} q_{1}^{3}+s_{21} q_{2}^{3}+s_{31} q_{1} q_{2}^{2}+s_{41} q_{1}^{2} q_{2}\right) \\
& \ddot{q}_{2}(\tau)+g_{2}^{2} q_{2}(\tau)=-\varepsilon \mu_{21} \dot{q}_{1}(\tau)-\varepsilon \mu_{22} \dot{q}_{2}(\tau)+\varepsilon\left(s_{12} q_{1}^{3}+s_{22} q_{2}^{3}+s_{32} q_{1} q_{2}^{2}+s_{42} q_{1}^{2} q_{2}\right)
\end{aligned}
$$

where $\varepsilon$ denotes a small parameter. The main coefficients are shown below, and the others are provided in Appendix I

$$
\begin{array}{ll}
q_{n}(\tau)=\frac{Q_{n}(t)}{l} & \tau=\omega_{n} t \quad \dot{q}_{1}(\tau)=\frac{\dot{Q}_{n}(t) l}{\omega_{n}} \\
\ddot{q}_{1}(\tau)=\frac{\ddot{Q}_{n}(t) l}{\omega_{n}^{2}} & \omega_{n}=p_{1}^{2} \sqrt{\frac{E I}{\rho A}}
\end{array}
$$




\subsection{Perturbation approach}

The displacement $w(x, t)$ is spread out into a combination of time and space variables. Adopting the assumed mode form of the first two order truncation in space, Eq. (3.2), so the nonlinear vibration equation, has been dispersed by the Galerkin method and rearranged by dimensionless processing from Eqs. (3.2)-(3.4). Finally, it can be solved by the multiple-scales method. The derivations of Eq. (3.5) are referred to the solution by the multiple-scales method ( $\mathrm{Li}$ et al., 2017; $\mathrm{Hu}$ and Wang, 2017). It is worth noting that the multi-scale method, a widely used but complex solving process, is a mature and an effective nonlinear solution theory (Nayfeh and Mook, 1979)

$$
\begin{aligned}
& a_{1}^{\prime}\left(T_{1}\right) g_{1}+\frac{1}{2} \mu_{11} a_{1}\left(T_{1}\right) g_{1}=\frac{1}{8} s_{41} a_{1}^{2}\left(T_{1}\right) a_{2}\left(T_{1}\right) \sin \gamma \\
& a_{2}^{\prime}\left(T_{1}\right) g_{2}+\frac{1}{2} \mu_{22} g_{2} a_{2}\left(T_{1}\right)=-\frac{1}{8} s_{12} a_{1}^{3}\left(T_{1}\right) \sin \gamma \\
& \gamma^{\prime}\left(T_{1}\right)=\sigma+\left(\frac{9 s_{11}}{8 g_{1}}-\frac{s_{42}}{4 g_{2}}\right) a_{1}^{2}\left(T_{1}\right)+\left(\frac{3 s_{31}}{4 g_{1}}-\frac{3 s_{22}}{8 g_{2}}\right) a_{2}^{2}\left(T_{1}\right) \\
& \quad+\left(\frac{3 s_{41} a_{1}\left(T_{1}\right) a_{2}\left(T_{1}\right)}{8 g_{1}}-\frac{s_{12} a_{1}^{3}\left(T_{1}\right)}{8 g_{2} a_{2}\left(T_{1}\right)}\right) \cos \gamma\left(T_{1}\right)
\end{aligned}
$$

where $a_{1}\left(T_{1}\right)$ and $a_{2}\left(T_{1}\right)$ denote the amplitudes of the first-order and second-order modes of the system, and $\gamma\left(T_{1}\right)$ denotes the phase angle. $g_{1}$ and $g_{2}$ denote the natural frequency of the first-order and second-order modes of the system, and $\sigma=\left(g_{2}-3 g_{1}\right) / \varepsilon$ is the tuning parameter of $g_{1}$ and $g_{2} . s_{i j}$ denote the coefficients of nonlinear terms (other definitions in the multiple-scales method are provided in Appendix I).

\subsection{System stability analysis}

After having done the work above, the next step is to find the solution to Eqs. (3.5). For the electromagnetically damped system, multiplying Eq. (3.5) $)_{1}$ by $g_{1}^{-1} a_{1}$ and Eq. $(3.5)_{2}$ by $g_{2}^{-1} \nu a_{2}$, where $\nu=s_{41} g_{2} /\left(s_{12} g_{1}\right)$, and adding the results together, we obtain

$$
a_{1}^{\prime} a_{1}+\nu a_{2}^{\prime} a_{2}=-\frac{1}{2} \mu_{11} a_{1}^{2}-\frac{1}{2} \nu \mu_{22} a_{2}^{2}
$$

Equation (3.6) can be integrated for a no electromagnetically damped system is

$$
a_{1}^{2}+\nu a_{2}^{2}=E
$$

where $E$ is a constant of integration, which is related to the initial energy of the system.

Changing the independent variable from $T_{1}$ to $a_{2}$ in Eq. (3.5) 3 , and using Eq. $(3.5)_{2}$, we obtain

$$
-a_{1}^{3} a_{2} \sin \gamma \frac{d \gamma}{d a_{2}}=\frac{8 g_{2} \sigma}{s_{12}} a_{2}+\lambda_{1} a_{2}^{3}+\lambda_{2} a_{1}^{2} a_{2}+\left(3 \nu a_{1} a_{2}^{2}-a_{1}^{3}\right) \cos \gamma
$$

where

$$
\lambda_{1}=\frac{6 g_{2} s_{31}}{g_{1} s_{12}}-\frac{3 s_{22}}{s_{12}} \quad \lambda_{2}=\frac{9 g_{2} s_{11}}{g_{1} s_{12}}-\frac{2 s_{42}}{s_{12}}
$$

Using Eq. (3.7) and the result of integration of Eq. (3.8), we are able to acquire

$$
a_{1}^{3} a_{2} \cos \gamma-\left(\frac{4 g_{2} \sigma}{s_{12}}+\frac{1}{2} \lambda_{2} E\right) a_{2}^{2}-\frac{1}{4}\left(\lambda_{2} \nu-\lambda_{1}\right) a_{2}^{4}=M
$$

where $M$ is a constant of integration. 
Setting $a_{1}^{2}=E \xi, a_{2}^{2}=E \nu^{-1}(1-\xi)$ and eliminating $\gamma$ from Eq. (3.5) 1 and Eq. (3.9), we obtain the following formula. At the same time, in order to simplify the expression, we introduce two new functions $F(\xi)$ and $G(\xi)$ (Nayfeh and Mook, 1979)

$$
\frac{16 \nu g_{1}^{2}}{E^{2} s_{41}^{2}}\left(\xi^{\prime}\right)^{2}=\xi^{2}(1-\xi)-\frac{G^{2} \nu}{E^{4}}=F^{2}(\xi)-G^{2}(\xi)
$$

where

$$
\begin{aligned}
& F(\xi)= \pm \sqrt{\xi^{2}(1-\xi)} \\
& G(\xi)=\frac{\sqrt{\nu}}{E^{2}}\left[L+\frac{E}{\nu}\left(\frac{4 g_{2} \sigma}{s_{12}}+\frac{1}{2} \lambda_{2} E\right)(1-\xi)+\frac{E^{2}}{4 \nu^{2}}\left(\lambda_{2} \nu-\lambda_{1}\right)(1-\xi)^{2}\right]
\end{aligned}
$$

To find the steady-state solutions of $a_{1}$ and $a_{2}$, we set $a_{1}^{\prime}=a_{2}^{\prime}=\gamma^{\prime}=0$ in Eqs. (3.5). Then, the steady-state solution without damping is given by

$$
\begin{aligned}
& \sin \gamma=0 \\
& a_{2} \sigma+\left(\frac{9 s_{11}}{8 g_{1}}-\frac{s_{42}}{4 g_{2}}\right) a_{1}^{2} a_{2}+\left(\frac{3 s_{31}}{4 g_{1}}-\frac{3 s_{22}}{8 g_{2}}\right) a_{2}^{3}+\left(\frac{3 s_{41} a_{1} a_{2}^{2}}{8 g_{1}}-\frac{s_{12} a_{1}^{3}}{8 g_{2}}\right) \cos n \pi=0
\end{aligned}
$$

\section{Numerical simulations}

The model of the simulation is supposed to be a conductive axially moving beam of a copper material. The main parameters are presented as follows: length of the beam is $l=0.3 \mathrm{~m}$, width of the beam is $b=0.02 \mathrm{~m}$, height of the beam is $h=0.01 \mathrm{~m}$, axial tension is $F_{0 x}=30000 \mathrm{~N}$, elastic modulus is $E=108 \mathrm{GPa}$, mass density is $\rho=8920 \mathrm{~kg} / \mathrm{m}^{3}$.

\subsection{System without electromagnetic damped}

\subsubsection{Vibration mode}

Based on the numerical solution to Eqs. (3.5) without electromagnetic damping, graphs of relevant vibration modes can be made as follows. Figures 2-5 describe vibration modes versus time for different initial conditions and system parameters. They all show the same phenomenon that the coupled first-order and second-order vibration modes change with time, which means the system energy constantly exchanges between different vibration modes because of internal resonance. On may find correlative descriptions about the internal resonance in the book by Nayfeh and Mook (1979).

Figures $2 \mathrm{a}-2 \mathrm{~d}$ show vibration modes versus time for different axial velocity and the initial conditions $a_{10}=a_{20}=0.05, \gamma_{0}=0$. They show that both the variation amplitudes and the intersections of vibration modes continuously grow up while the axial velocity $c$ increases from $5 \mathrm{~m} / \mathrm{s}$ to $95 \mathrm{~m} / \mathrm{s}$.

Figures 3-5 describe vibration modes versus time for different initial conditions when and the axial velocity $c=5 \mathrm{~m} / \mathrm{s}$. And the primary distinction between them is the emphasis on effects of different parameters taken into account, in which the first picture focuses on the influence of the initial value $\gamma_{0}$, and the other two care about $a_{0}$.

As the initial value of the phase angle $\gamma_{0}$ moves on, the first-order and the second-order modes have different manifestations, the former is overall upward and the latter goes down integrally, see Fig. 3. In addition, the curves of vibration modes are intertwined when $0<\gamma_{0}<9.4$, but they will separate when $\gamma_{0}$ goes beyomd that range.

Then, we investigate the influence of the initial value $a_{0}$ on vibration modes when taking $\gamma_{0}=0$ and $\gamma_{0}=9.4$, respectively. As shown in Figs. 4 and 5, the amplitude changes of the 
(a)

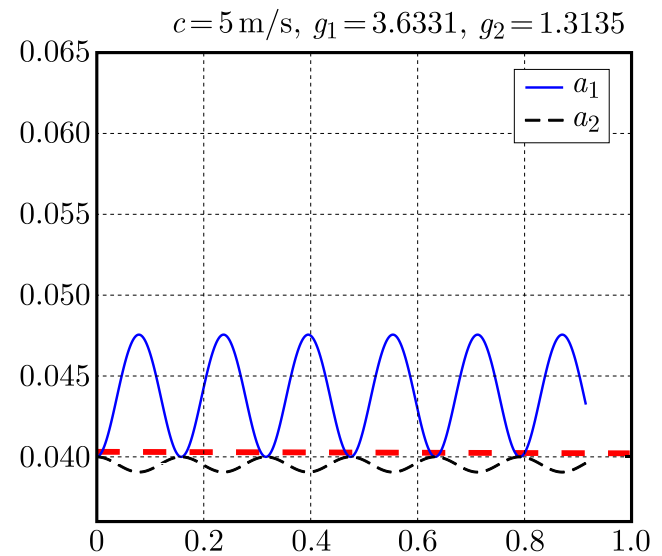

(c)

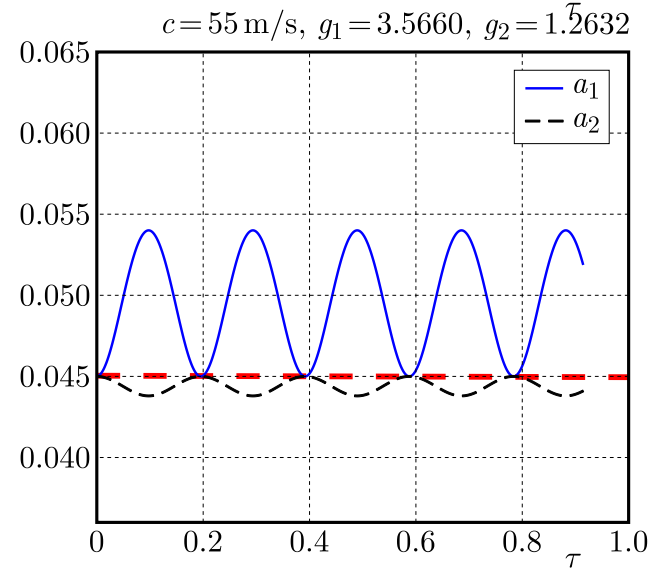

(b)

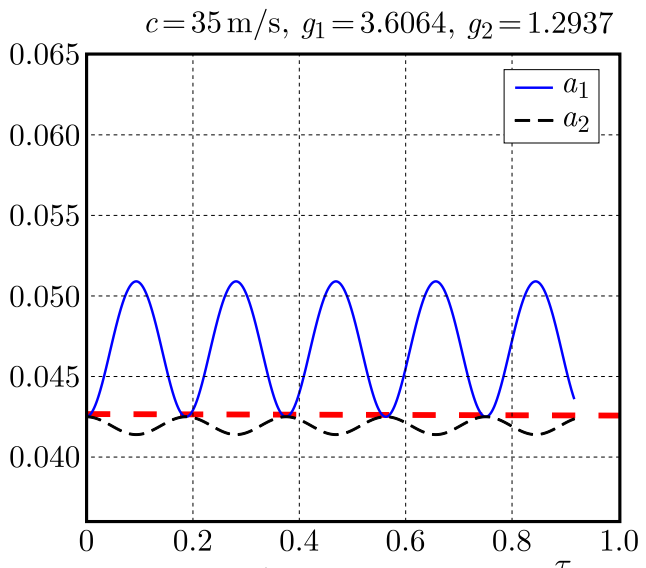

(d)

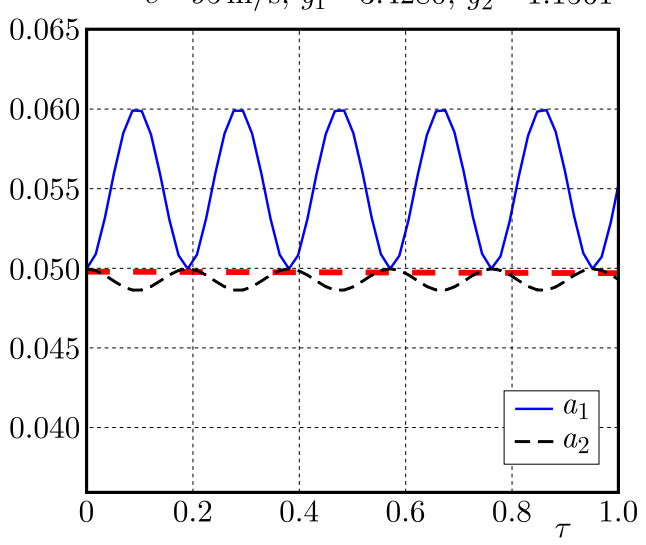

Fig. 2. Variation modes for the axial velocity $c\left(a_{10}=a_{20}=0.05, \gamma_{0}=0\right)$. The letters $g_{1}$ and $g_{2}$ represent natural frequencies of the first-order and second-order vibration modes

(a)

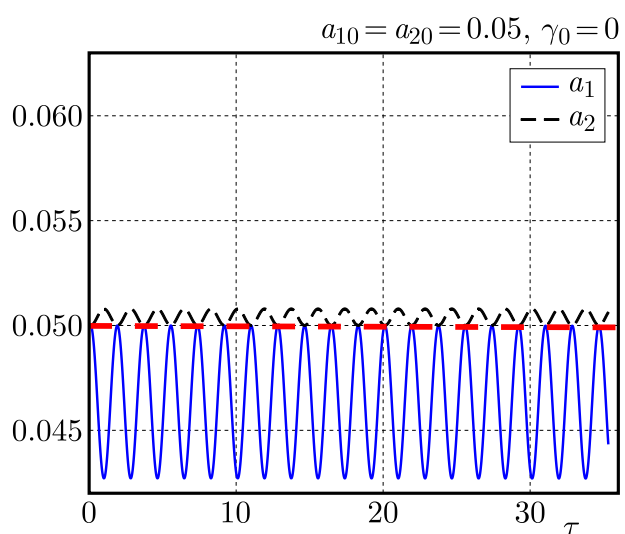

(c)

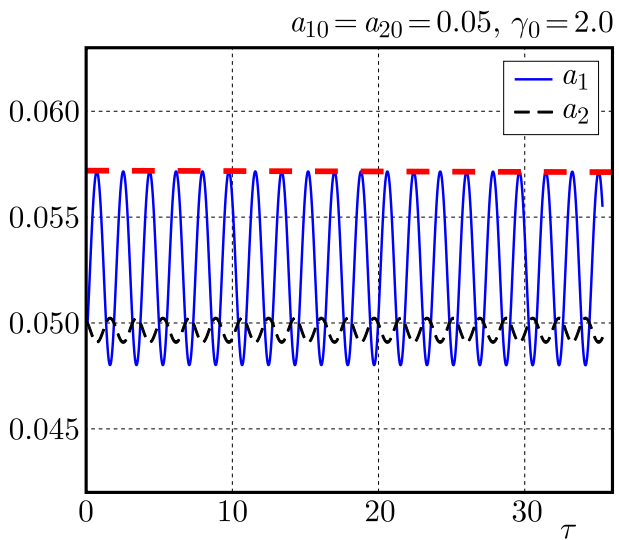

(b)

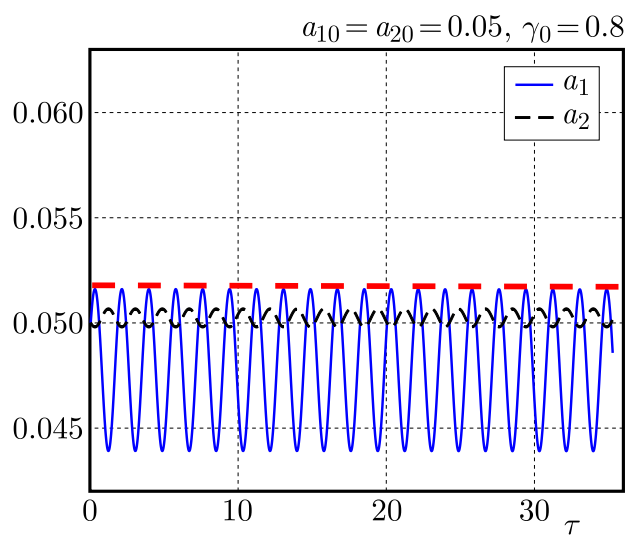

(d)

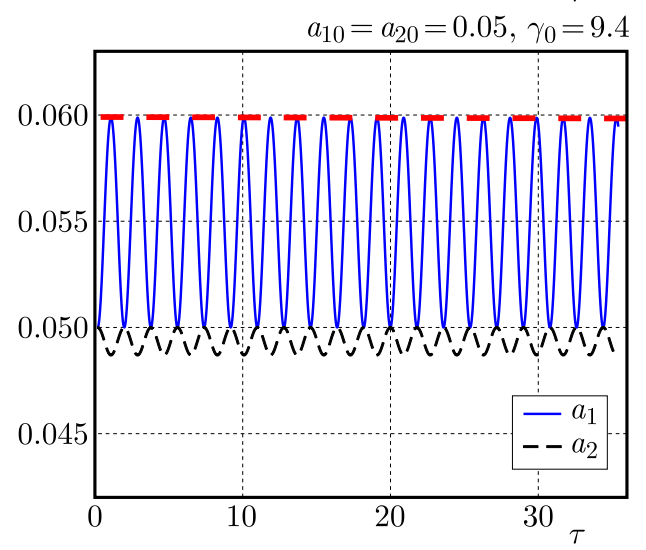

Fig. 3. Variation modes for different initial conditions $\gamma_{0}\left(a_{0}=0.05, c=55 \mathrm{~m} / \mathrm{s}\right)$ 
(a)

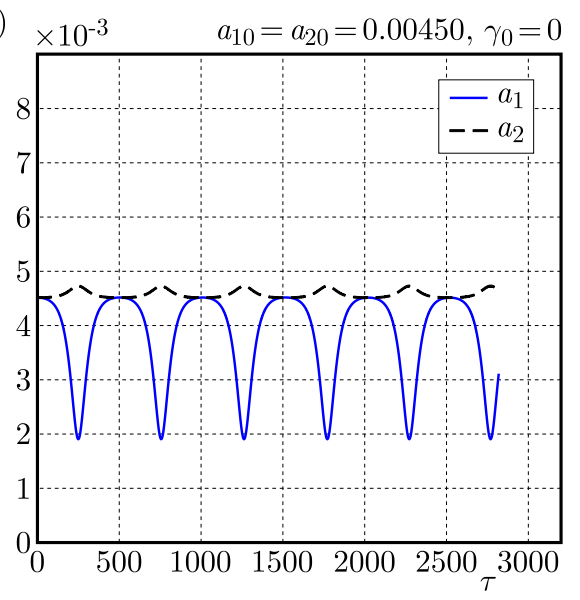

(c)

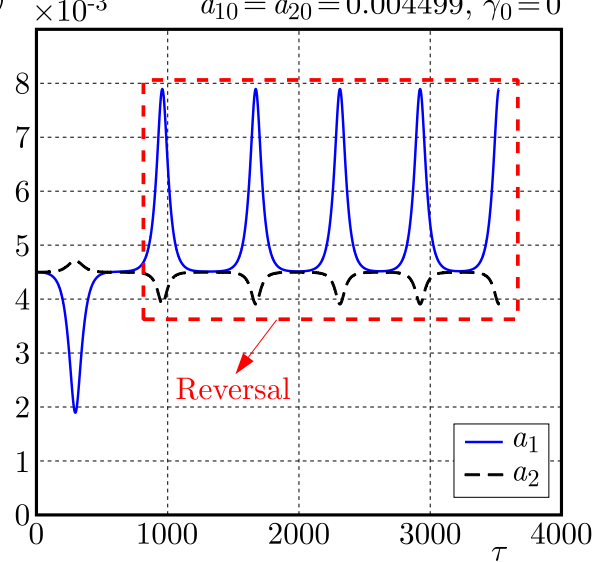

(b)

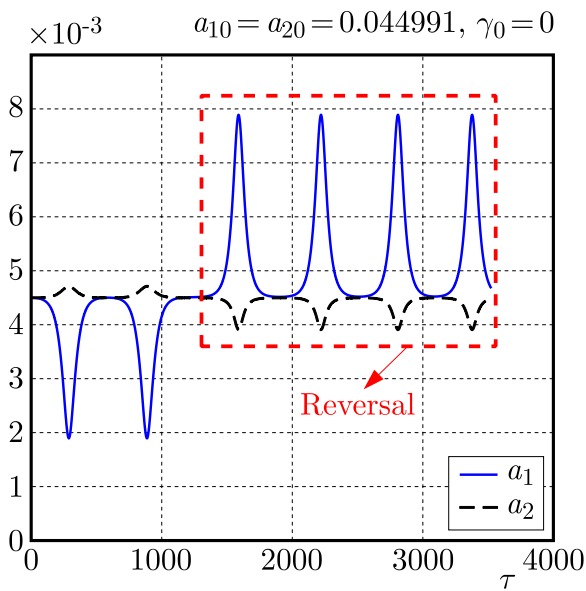

(d)

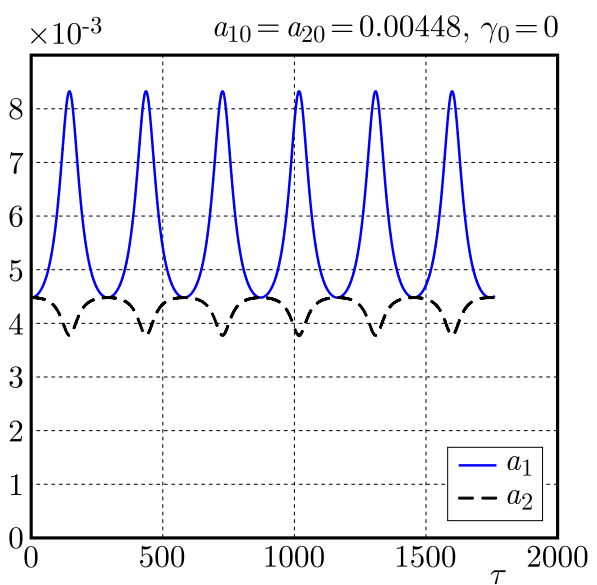

Fig. 4. Variation modes for different initial conditions $a\left(\gamma_{0}=0, c=55 \mathrm{~m} / \mathrm{s}\right)$

(a)

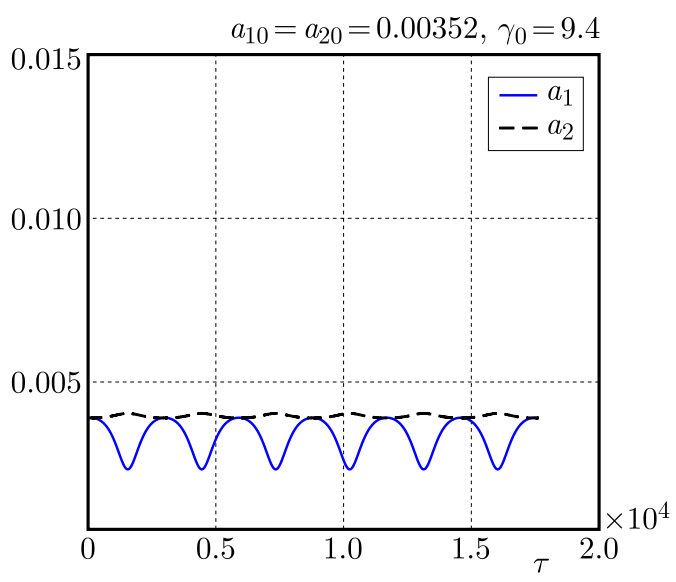

(c)

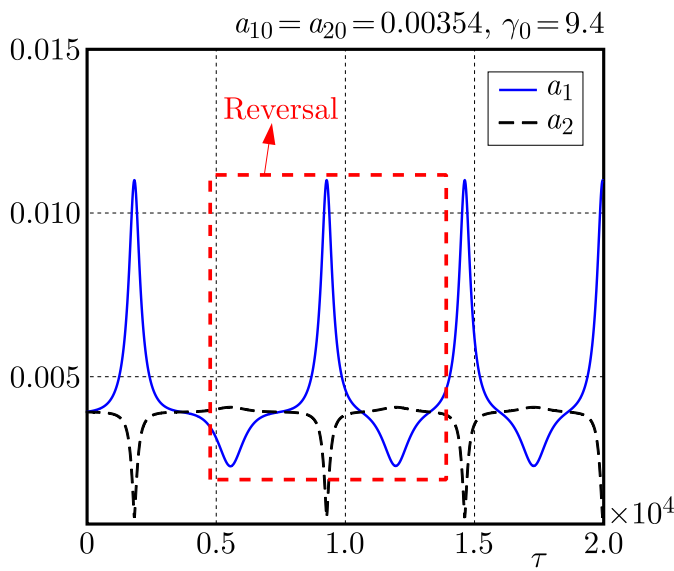

(b)

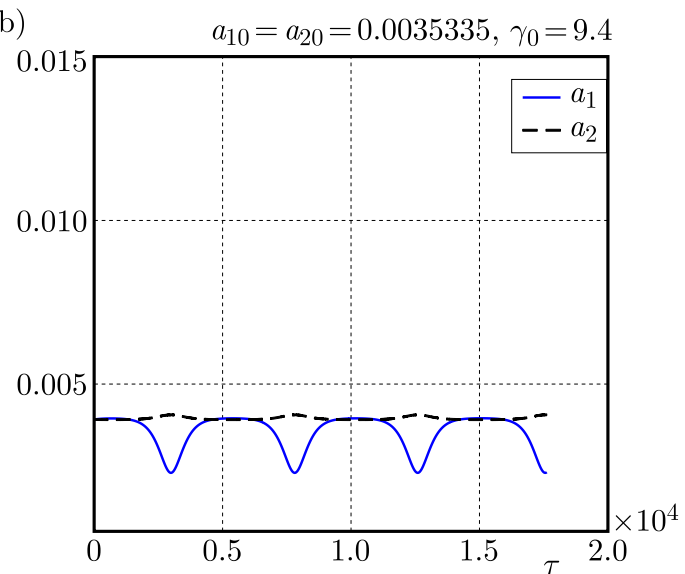

(d)

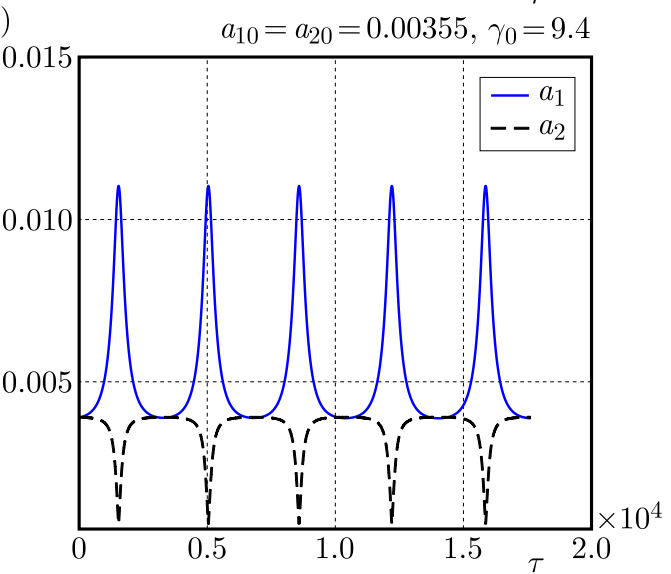

Fig. 5. Variation modes for different initial conditions $a\left(\gamma_{0}=9.4, c=55 \mathrm{~m} / \mathrm{s}\right)$ 
first-order and second-order mode are very special, similar to "reversal behavior" in Figs. 4b,c and Figs. 5b,c rather than the whole moving up and down in Fig. 3. From the contrast between Figs. 3, 4 and 5, we can conclude that the vibration modes are more sensitive to the initial amplitude of the vibration mode $a_{0}$ than the phase angle $\gamma_{0}$.

Subsequently, we have carried out direct simulations of equations Eq. (3.3) in order to evaluate the accuracy of the perturbation approach. When using different values of the initial conditions, we obtained time-history graphs, phase-plane diagrams and Poincaré maps of the vibration amplitudes $q_{1}$ and $q_{2}$.

Figures 6, 7 and 8 demonstrate the responses of the system for different initial conditions $a_{10}=a_{20}=q_{10}=q_{20}=0.00055, a_{10}=a_{20}=q_{10}=q_{20}=0.005$ and $a_{10}=a_{20}=q_{10}=$ $q_{20}=0.012$. Compared to the amplitudes $a_{1}$ and $a_{2}$ from the perturbation approach depicted in (a) of Figs. 6-8, the numerical amplitudes $q_{1}$ and $q_{2}$ displayed in (b) of Figs. 6-8 present good consistency. Besides, Figs. (c), (d) and (e), (f) describe the corresponding phase diagram and Poincaré map of $q_{1}$ and $q_{2}$. It can be clearly seen that the responses of the system firstly change from the periodic motion to period-2 motion and, finally, develop into chaotic motion when the initial conditions $q_{10}$ and $q_{20}$ increase.
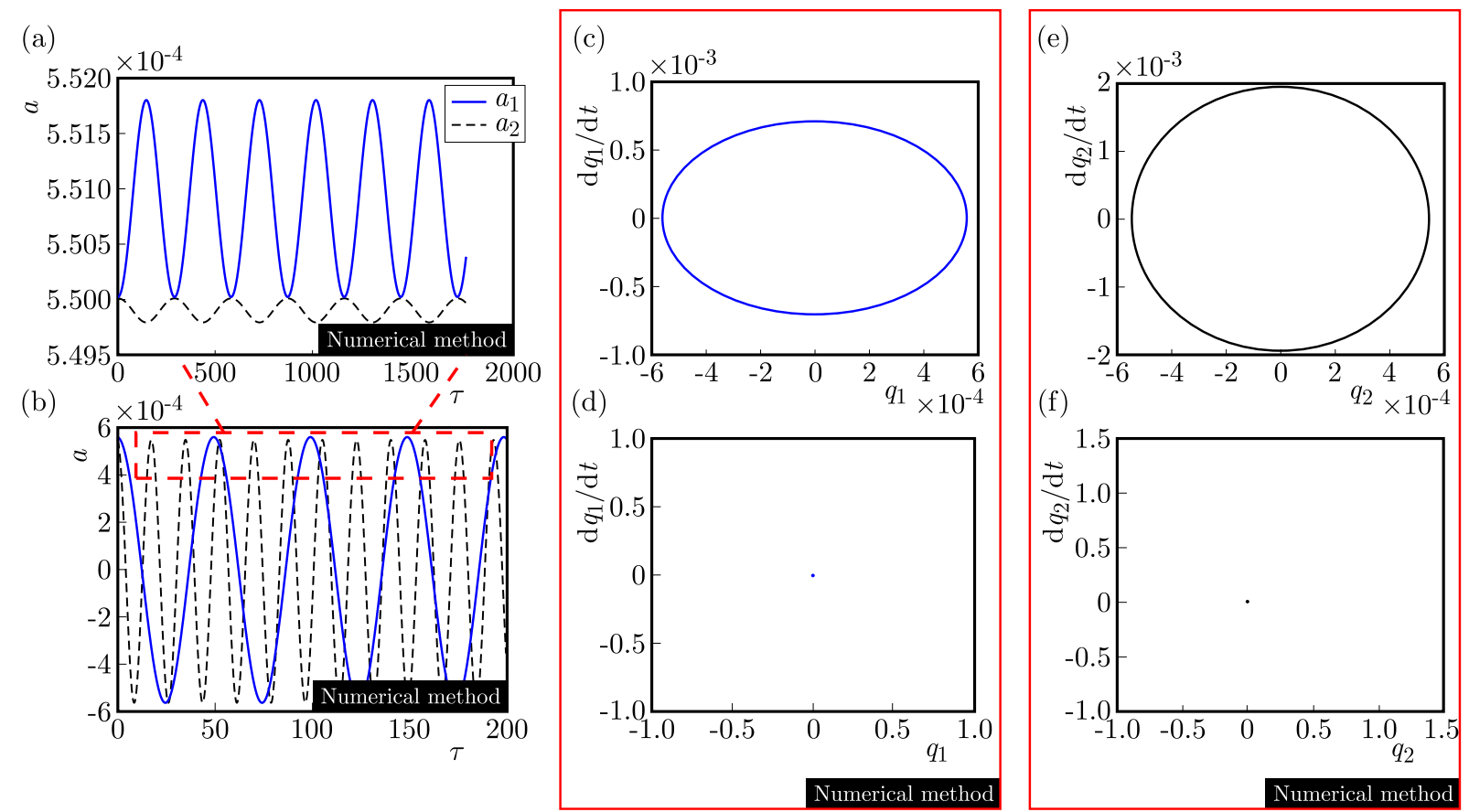

Fig. 6. Periodic motion of the system for the initial condition equal to $0.00055\left(\gamma_{0}=0, c=55 \mathrm{~m} / \mathrm{s}\right)$;

(c) and (e) are phase diagrams of $q_{1}$ and $q_{2},(\mathrm{~d})$ and (f) are Poincaré maps of $q_{1}$ and $q_{2}$

When we calculate and analyze the internal resonance of the system without electromagnetic damping, the functions of $F(\xi)$ and $G(\xi)$ in Eq. (3.11) are plotted in Fig. 9a. Since $\xi$ and $a$ must be real, $F^{2}(\xi) \geqslant G^{2}(\xi)$. The points where $G(\xi)$ meets $F(\xi)$ correspond to $\xi^{\prime}=0$. It also means that the vibration modes $a_{1}^{\prime}=a_{2}^{\prime}=0$. The curve $G_{3}$ which has two different crossing points with $F$ corresponds to the steady-state solution of $\xi$ and, hence, $a_{1}$ and $a_{2}$. The points such as $P_{1}$, where $G_{2}$ touches $F$ and $P_{2}$, where $G_{4}$ touches $F$ represent the unique steady-state solution of $\xi$. On the other hand, Fig. 4 shows curves like $G_{1}$ and $G_{5}$ meeting $F$ at no point, which means that there is no steady-state solution in the system. In addition, we should note that the steady-state motions are not always stable because any small outer disturbance would lead to the curves $G_{2}$ and $G_{4}$ similar to other curves. Figure 9b shows characteristic graphs of the vibration amplitude for different axial velocity. As shown in Fig. 9b, with an increase in the axial velocity, the characteristic curve circle gradually narrows down and moves towards the origin. 
(a)

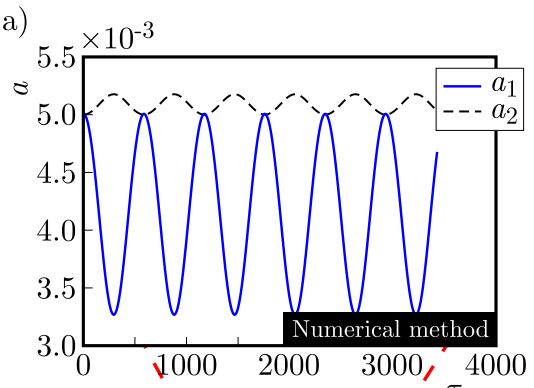

(b)

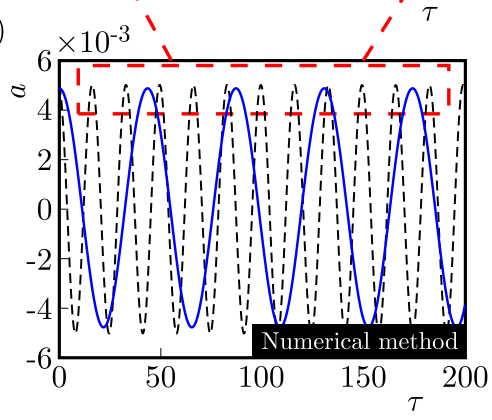

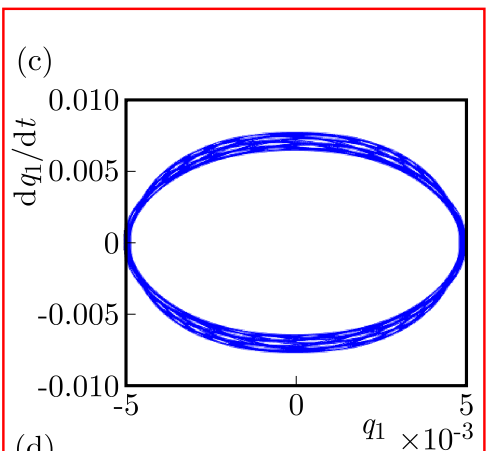

(d)

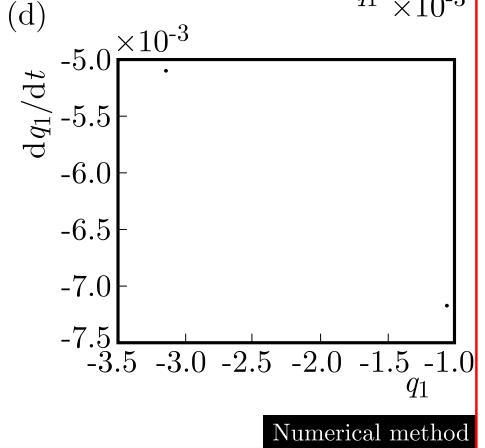

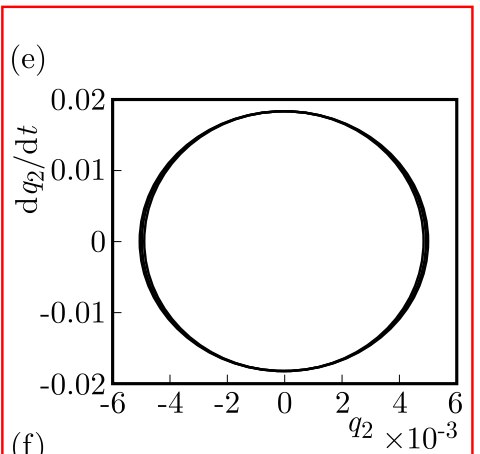

(f)

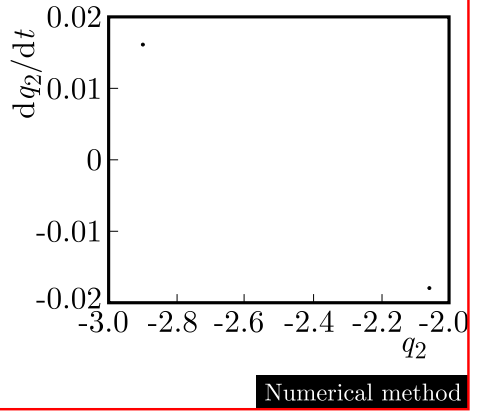

Fig. 7. Periodic motion of the system for the initial condition equal to $0.005\left(\gamma_{0}=0, c=55 \mathrm{~m} / \mathrm{s}\right)$;

(c) and (e) are phase diagrams of $q_{1}$ and $q_{2}$, (d) and (f) are Poincaré maps of $q_{1}$ and $q_{2}$

(a)

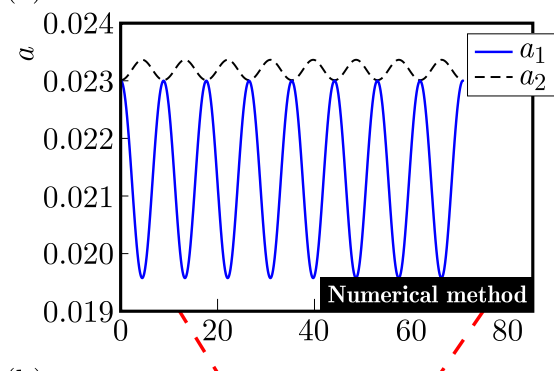

(b)

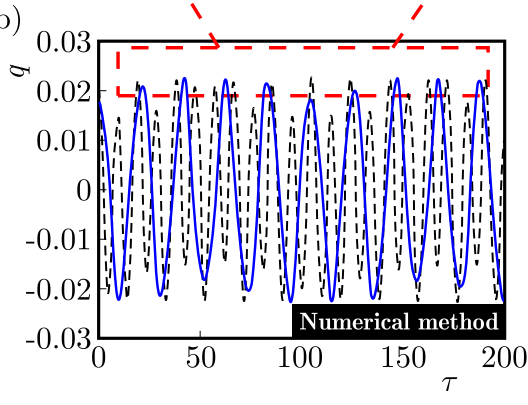

(c)

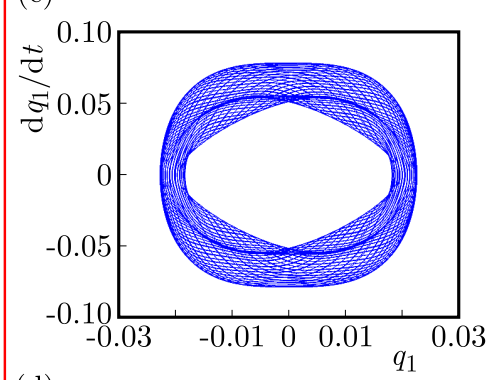

(d)

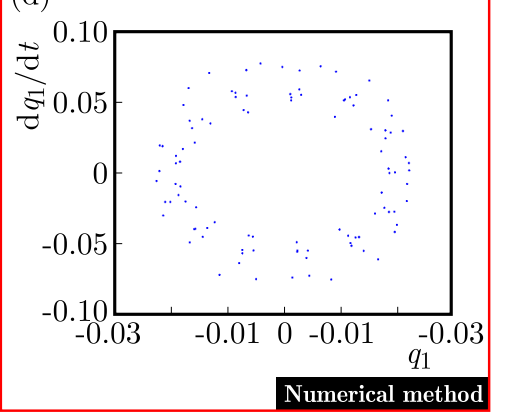

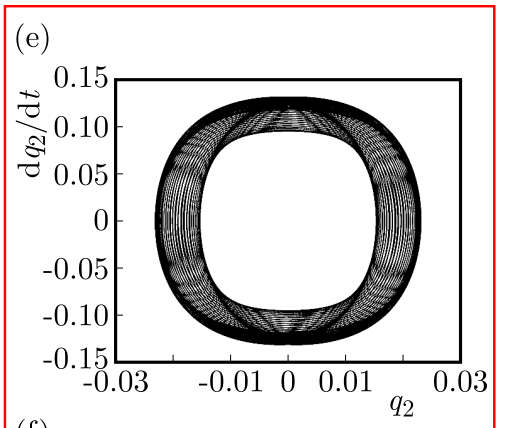

(f)

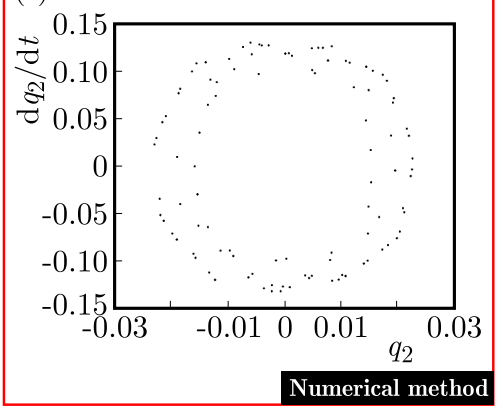

Fig. 8. Periodic motion of the system for the initial condition equal to $0.012\left(\gamma_{0}=0, c=55 \mathrm{~m} / \mathrm{s}\right)$;

(c) and (e) are phase diagrams of $q_{1}$ and $q_{2}$, (d) and (f) are Poincaré maps of $q_{1}$ and $q_{2}$

\subsubsection{Electromagnetically damped system}

Similarly, basing on the numerical solution to Eqs. (3.5) in the damped free vibration system, we obtain the following decay graphs of vibration modes. 
(a)

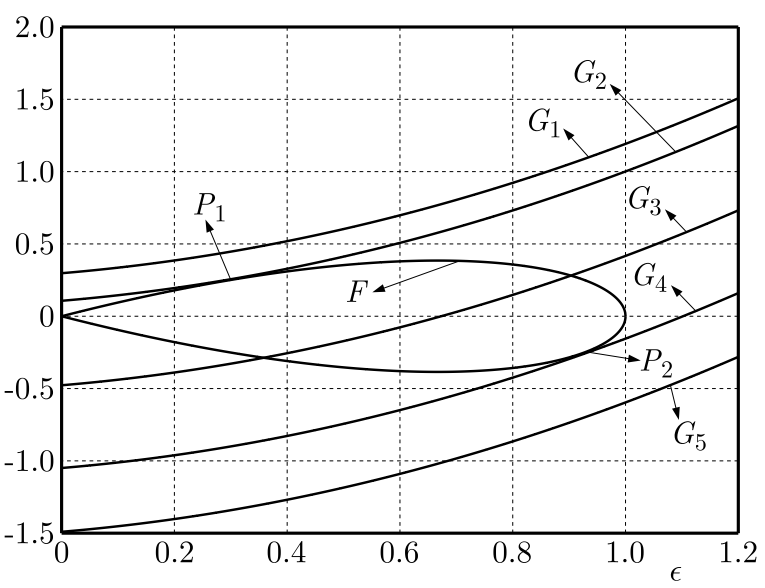

(b)

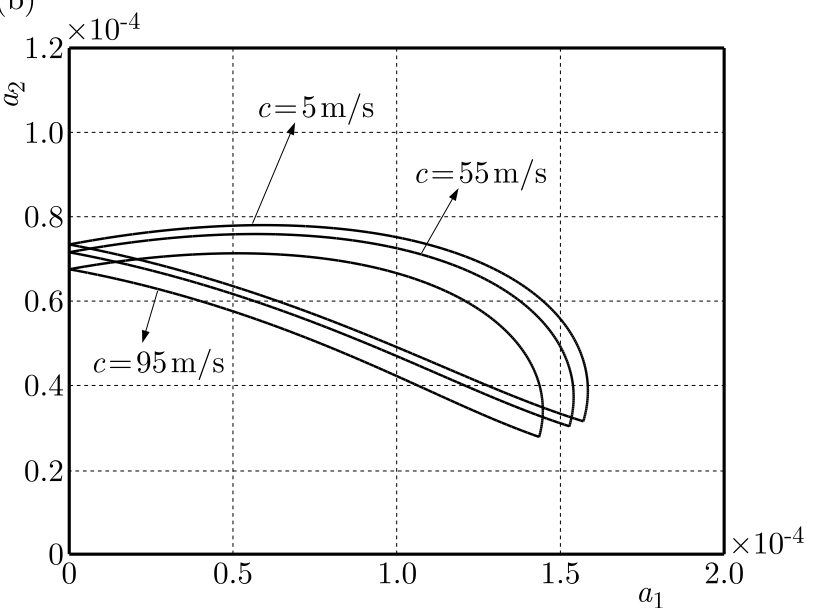

Fig. 9. (a) The curve graph of $F$ and $G$. (b) The characteristic graphs of the vibration amplitude $(\sin \gamma=0)$

\subsection{Electromagnetic damped system}

Figure 10a reveals the decay graphs of vibration modes for different axial velocities $(c=$ $5 \mathrm{~m} / \mathrm{s}, c=55 \mathrm{~m} / \mathrm{s}, c=95 \mathrm{~m} / \mathrm{s})$. It should be observed that the axial velocity may exert a more significant effect on the first-order mode than on the second one. Figure 10b exhibits the decay graphs of vibration modes for different initial conditions $a_{10}=0.04,0.05$ and 0.06 , as a result of which their attenuation trend is almost the same, but the difference lies in that the larger the initial value is, the more slender the waveforms of vibration mode are.

(a)

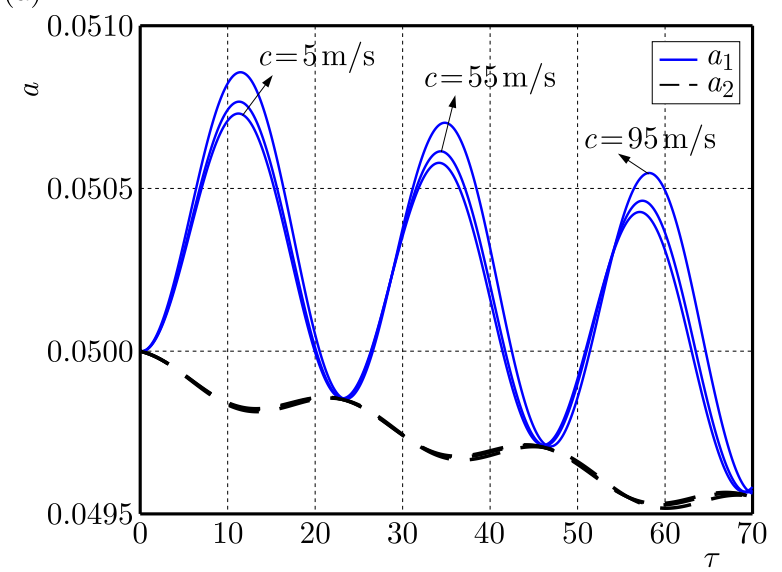

(b)

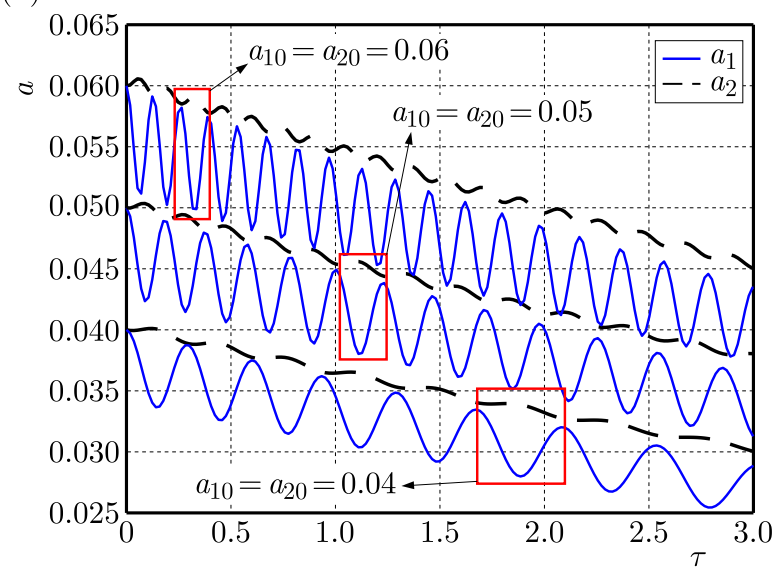

Fig. 10. The decay graphs of variation modes for different axial velocities and different initial conditions: (a) $B_{0 y}=0.3 \mathrm{~T}, \gamma=9.4$ and (b) $B_{0 y}=0.3 \mathrm{~T}, \gamma=0$

Figures 11a-h show the decay graphs of vibration modes for different magnetic field strength in the damped free vibration system. Graphs (a), (c), (e), (g) of vibration modes $a_{1}$ and $a_{2}$, at the bottom of Fig. 11, are calculated by the perturbation approach, and curves (b), (d), (f), (h) of $q_{1}$ and $q_{2}$, at the bottom of Fig. 11, are drawn from direct simulations of vibration equations for the magnetic field strength $B_{0 y}=0.1 \mathrm{~T}, B_{0 y}=0.3 \mathrm{~T}, B_{0 y}=0.6 \mathrm{~T}, B_{0 y}=1.2 \mathrm{~T}$, respectively. Thus, as reported by Nayfeh and Mook (1979) on the internal resonance phenomenon in the damped system, the curves of vibration modes in our investigation exhibit the same trend of coupled attenuation with time. Their decay rate will increase when the applied magnetic field becomes stronger. 

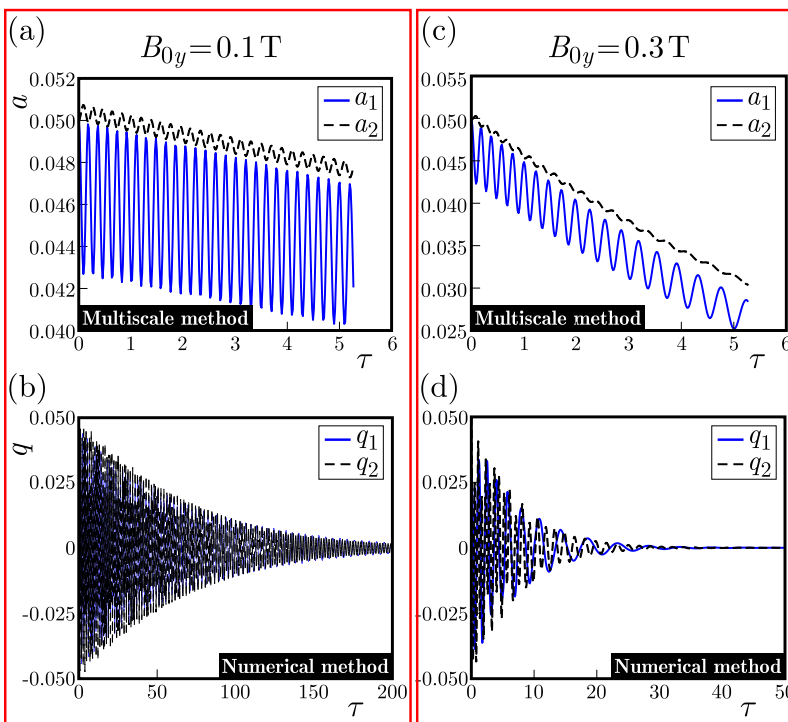

(d)

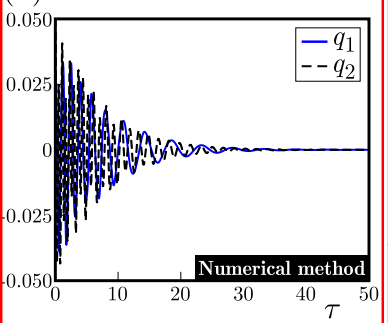

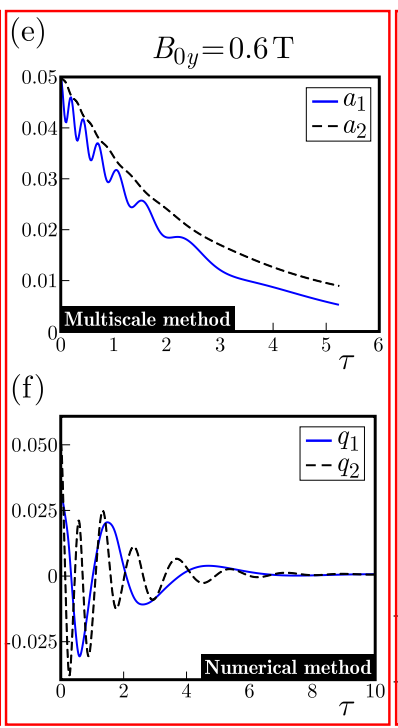

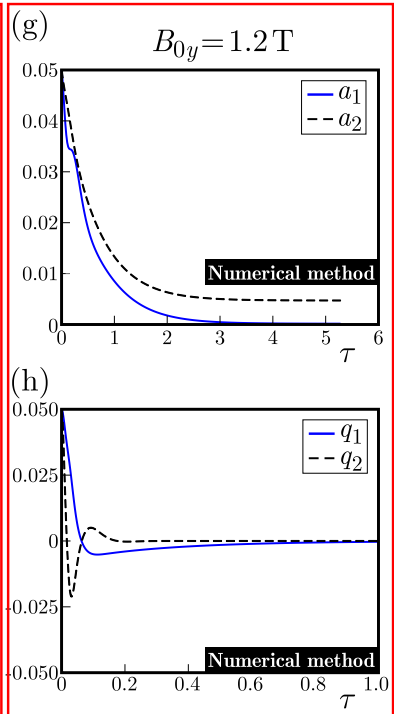

Fig. 11. The system attenuation response for different magnetic field intensity (multistate and numerical methods)

\section{Conclusions}

This work is mainly concerned with the 1:3 internal resonance problem of an axially moving conducting elastic beam in a magnetic field. Based on our study, the magneto-elastic vibration equation of the beam can be obtained and the vibration mode equations of free vibrating beams can be obtained through the multi-scale method. Meanwhile, we have carried out direct simulations of the vibration equations in order to evaluate the accuracy of the perturbation approach.

- In the system without electromagnetic damping, there are steady-state motions and solutions, where the system energy is constantly exchanged between the first two coupled vibration modes. The steady-state motion, however, is not always stable. As shown in Fig. 9a, any small external disturbance can cause changes. In addition, the vibration amplitudes continuously increase while the axial velocity $c$ enhances from $5 \mathrm{~m} / \mathrm{s}$ up to $95 \mathrm{~m} / \mathrm{s}$. As for the initial conditions, the amplitude changes of the first-order and second-order modes resulting from an increase in the initial amplitude of vibration modes $a_{10}$ and $a_{20}$ (see Appendix I). They are displayed in Figs. 4b,c and Figs. 5b,c. And unlike the whole up or down motions caused by the increase of the initial phase angle $\gamma_{0}$ (see Appendix I) shown in Fig. 3, they present "reversal behaviour". The vibration modes are more sensitive to the initial amplitude of vibration modes, and the curves of vibration modes are intertwined when the initial condition satisfies $0<\gamma_{0}<9.4, a_{10}=a_{20}=0.05$ but they separate for phase angles beyond that range.

- Direct simulations of the vibration equations have been carried out in order to evaluate the accuracy of the perturbation approach. Compared to the amplitudes from the perturbation approach, see Figs. 6-8a, the amplitudes displayed in Figs. 6-8b present good consistency. Meanwhile, the corresponding phase diagram and Poincaré map of the system are obtained, which show that the responses of the system change firstly from the periodic motion to period-2 motion and, finally, develop into chaotic motion when the initial conditions increase.

- For the electromagnetically damped system, the vibration modes exhibit a trend of coupling attenuation with time. Its decay rate increases when the applied magnetic field becomes stronger. Additionally, the axial velocity may exert a more significant effect on 
the first-order mode than on the second one. As for the initial amplitude of vibration modes, the larger the initial value is, the slenderer the waveforms of vibration modes seem to be.

\section{Acknowledgement}

This research was supported by the National Natural Science Foundation of China (No. 11472239), Hebei Provincial Natural Science Foundation of China (No. A2015203023) and the Key Project of Science and Technology Research of Higher Education of Hebei Province of China (No. ZD20131055).

\section{Appendix I}

Coefficients of Eq. (3.3) $(n=1,2 ; i=1,2)$

$$
\begin{aligned}
& A_{n i}=\int_{0}^{l} X_{n} X_{i} d x B_{n i}=\int_{0}^{l} \frac{d X_{n}}{d x} X_{i} d x \\
& C_{n i}=\int_{0}^{l} \frac{d^{2} X_{n}}{d x^{2}} X_{i} d x D_{n i}=\int_{0}^{l} \frac{d^{4} X_{n}}{d x^{4}} X_{i} d x \\
& S_{1 i}=\int_{0}^{l} \frac{d^{2} X_{1}}{d x^{2}}\left(\frac{d X_{1}}{d x}\right)^{2} X_{i} d x S_{3 i}=\int_{0}^{l}\left[\frac{d^{2} X_{1}}{d x^{2}}\left(\frac{d X_{2}}{d x}\right)^{2}+2 \frac{d X_{1}}{d x} \frac{d X_{2}}{d x} \frac{d^{2} X_{2}}{d x^{2}}\right] X_{i} d x \\
& S_{2 i}=\int_{0}^{l}\left(\frac{d X_{2}}{d x}\right)^{2} \frac{d^{2} X_{2}}{d x^{2}} X_{i} d x S_{4 i}=\int_{0}^{l}\left[\left(\frac{d X_{1}}{d x}\right)^{2} \frac{d^{2} X_{2}}{d x^{2}}+2 \frac{d^{2} X_{1}}{d x^{2}} \frac{d X_{1}}{d x} \frac{d X_{2}}{d x}\right] X_{i} d x \\
& D_{n i}=P_{n}^{4} A_{n 1}=0 \quad A_{i j}=C_{i j}=D_{i j}=0
\end{aligned}
$$

Coefficients of Eqs. (3.4) $(n=1,2 ; i=1,2,3,4)$

$$
\begin{aligned}
& g_{1}^{2}=\frac{C_{11}}{A_{11}} \eta^{2}-\frac{C_{11}}{A_{11}} \zeta+1 \quad g_{2}^{2}=\frac{C_{22}}{A_{22}} \eta^{2}-\frac{C_{22}}{A_{22}} \zeta+\left(\frac{9}{5}\right)^{4} \quad \eta=\frac{c}{\omega_{n}} \\
& \zeta=\frac{F_{0 x}}{\rho A \omega_{n}^{2}} \quad \mu_{11}=\mu_{22}=\frac{1}{\varepsilon} \frac{\sigma B_{0 y}^{2}}{2 \rho \omega_{n}} \quad \mu_{12}=\mu_{21}=\frac{1}{\varepsilon} \frac{2 B_{21} \eta}{A_{11}} \\
& k_{1}=\frac{18 l^{2}}{D_{11} h^{2}} \quad k_{2}=\left(\frac{9}{5}\right)^{4} \frac{18 l^{2}}{D_{22} h^{2}} \quad s_{i 1}=k_{1} \frac{S_{i 1}}{\varepsilon} \quad s_{i 2}=k_{2} \frac{S_{i 2}}{v e}
\end{aligned}
$$

Definitions in multiple-scales method of Eqs. (3.5)

$$
\begin{array}{lc}
q_{1}(\tau, \varepsilon)=q_{11}\left(T_{0}, T_{1}\right)+\varepsilon q_{12}\left(T_{0}, T_{1}\right) & q_{2}(\tau, \varepsilon)=q_{21}\left(T_{0}, T_{1}\right)+\varepsilon q_{22}\left(T_{0}, T_{1}\right) \\
q_{11}=A_{1}\left(T_{1}\right) \mathrm{e}^{\mathrm{i} g_{1} T_{0}}+\bar{A}_{1}\left(T_{1}\right) \mathrm{e}^{\mathrm{i} g_{1} T_{0}} & q_{21}=A_{2}\left(T_{1}\right) \mathrm{e}^{\mathrm{i} g_{2} T_{0}}+\bar{A}_{2}\left(T_{1}\right) \mathrm{e}^{\mathrm{i} g_{2} T_{0}}
\end{array}
$$

where $\bar{A}$ is the conjugate of $A$, i is the imaginary unit.

$$
A_{n}\left(T_{1}\right)=\frac{1}{2} a_{n}\left(T_{1}\right) \mathrm{e}^{\mathrm{i} \beta_{n}\left(T_{1}\right)} \quad n=1,2 \quad \gamma\left(T_{1}\right)=\beta_{2}\left(T_{1}\right)-3 \beta_{1}\left(T_{1}\right)+\sigma T_{1}
$$

where $T_{0}=\tau$ and $T_{1}=\varepsilon \tau$ are the time scales. 


\section{References}

1. Chen L.Q., TAng Y.Q., Lim C.W., 2010, Dynamic stability in parametric resonance of axially accelerating viscoelastic Timoshenko beams, Journal of Sound and Vibration, 329, 5, 547-565

2. Ding H., Chen L.Q., 2010, Galerkin methods for natural frequencies of high-speed axially moving beams, Journal of Sound and Vibration, 329, 17, 3484-3494

3. Hu Y., Hu P., Zhang J., 2015, Strongly nonlinear subharmonic resonance and chaotic motion of axially moving thin plate in magnetic field, Journal of Computational and Nonlinear Dynamics, 10, 2, 021010

4. Hu Y., WANG J., 2017, Principal-internal resonance of an axially moving current-carrying beam in magnetic field, Nonlinear Dynamics, 90, 1, 683-695

5. Li J., Hu Y.D., WANG Y.N., 2017, The magneto-elastic internal resonances of rectangular conductive thin plate with different size ratios, Journal of Mechanics, 34, 5, 711-723

6. Mao X.Y., Ding H., Chen L.Q., 2016, Steady-state response of a fluid-conveying pipe with 3:1 internal resonance in supercritical regime, Nonlinear Dynamics, 86, 2, 795-809

7. Mao X.Y., Ding H., Lim C.W., Chen L.Q., 2016, Super-harmonic resonance and multifrequency responses of a super-critical translating beam, Journal of Sound and Vibration, 385, $267-283$

8. NAyfeh A.H., Mook D.T., 1979, Nonlinear Oscillation, John Wiley \& Sons, New York

9. Pellicano F., 2005, On the dynamic properties of axially moving systems, Journal of Sound and Vibration, 281, 3-5, 593-609

10. Pratiher B., 2011, Non-linear response of a magneto-elastic translating beam with prismatic joint for higher resonance conditions, International Journal of Non-Linear Mechanics, 46, 5, 685-692

11. Pratiher B., Dwivedy S.K., 2009, Non-linear dynamics of a soft magneto-elastic Cartesian manipulator, International Journal of Non-Linear Mechanics, 44, 7, 757-768

12. Sahoo B., Panda L.N., Pohit G., 2015, Two-frequency parametric excitation and internal resonance of a moving viscoelastic beam, Nonlinear Dynamics, 82, 4, 1721-1742

13. Sahoo B., Panda L.N., Pohit G., 2016, Combination, principal parametric and internal resonances of an accelerating beam under two frequencies parametric excitation, International Journal of Non-Linear Mechanics, 78, 35-44

14. TAng Y.Q., Zhang D.B., Gao J.M., 2016, Parametric and internal resonance of axially accelerating viscoelastic beams with the recognition of longitudinally varying tensions, Nonlinear Dynamics, 83, 1-2, 401-418

15. Wang L., Chen H.H., He X.D., 2011, Active H control of the vibration of an axially moving cantilever beam by magnetic force, Mechanical Systems and Signal Processing, 25, 8, 2863-2878

16. Wu G.Y., 2007, The analysis of dynamic instability on the large amplitude vibrations of a beam with transverse magnetic fields and thermal loads, Journal of Sound and Vibration, 302, 1-2, $167-177$

17. Yan Q., Ding H., Chen L., 2015, Nonlinear dynamics of axially moving viscoelastic Timoshenko beam under parametric and external excitations, Applied Mathematics and Mechanics, 36, 8, 971-984 